\title{
Protocol for understanding acute sarcopenia: a cohort study to characterise changes in muscle quantity and physical function in older adults following hospitalisation
}

Carly Welch ${ }^{1,2,3,4^{*}}$ (D), Carolyn A. Greig ${ }^{1,5,6,7}$, Tahir Masud ${ }^{1,8,9,10}$, Thomas Pinkney ${ }^{3,11}$ and Thomas A. Jackson 1,2,3,12

\begin{abstract}
Background: Older adults are vulnerable to the effects of acute sarcopenia (acute muscle insufficiency) following hospitalisation. However, this condition remains poorly characterised to date. It is hypothesised that acute sarcopenia arises due to a combination of bed rest and inflammatory surge. This study aims to characterise changes in muscle quantity and function, determining which factors (clinical and biological) are most predictive, and how these relate to change in physical function at 13 weeks.

Methods: This study will include three groups of patients aged 70 years and older; patients undergoing elective colorectal surgery, patients admitted for emergency abdominal surgery, and patients admitted under general medicine with acute bacterial infections. Changes in muscle quantity (Bilateral Anterior Thigh Thickness with ultrasound and bioelectrical impedance analysis) and muscle function (muscle strength, physical performance) within 1 week of hospitalisation or surgery will be characterised, with follow-up of patients at 13 weeks. Physical function will be measured using the Patient Reported Outcome Measures Information System, and the Short Physical Performance Battery (or gait speed alone within 1 week of surgery).
\end{abstract}

Discussion: This study will fully characterise changes in muscle quantity and function in hospitalised older adults and enable risk stratification towards targeted interventions in clinical practice. The results of this study will inform further research involving interventions to ameliorate changes.

Trial registration: ClinicalTrials.gov Identifier: NCT03858192; Prospectively registered 28th February 2019.

Keywords: Acute sarcopenia, Physical function, Older adults, Hospitalisation

\footnotetext{
* Correspondence: c.welch@bham.ac.uk

${ }^{1}$ Medical Research Council and Versus Arthritis Centre for Musculoskeletal

Ageing Research, University of Birmingham and University of Nottingham, Birmingham and Nottingham, UK

${ }^{2}$ Institute of Inflammation and Ageing, University of Birmingham, Birmingham, UK

Full list of author information is available at the end of the article
}

(c) The Author(s). 2020 Open Access This article is licensed under a Creative Commons Attribution 4.0 International License, which permits use, sharing, adaptation, distribution and reproduction in any medium or format, as long as you give appropriate credit to the original author(s) and the source, provide a link to the Creative Commons licence, and indicate if changes were made. The images or other third party material in this article are included in the article's Creative Commons licence, unless indicated otherwise in a credit line to the material. If material is not included in the article's Creative Commons licence and your intended use is not permitted by statutory regulation or exceeds the permitted use, you will need to obtain permission directly from the copyright holder. To view a copy of this licence, visit http://creativecommons.org/licenses/by/4.0/ The Creative Commons Public Domain Dedication waiver (http://creativecommons.org/publicdomain/zero/1.0/) applies to the data made available in this article, unless otherwise stated in a credit line to the data. 


\section{Background}

Acute sarcopenia is an emerging condition of acute muscle insufficiency; older adults are considered particularly vulnerable to its effects following hospitalisation [1]. The European Working Group on Sarcopenia in Older People 2 (EWGSOP2) defines sarcopenia as reduced muscle strength with reduced muscle quantity or quality; cut-off values to meet criteria are usually set 2.0-2.5 standard deviations below the mean of a young adult healthy reference population. Additional demonstration of low physical performance is defined as severe sarcopenia. The revised definition (EWGSOP2) includes a distinction between acute and chronic sarcopenia; acute sarcopenia is defined as incident sarcopenia within 6 months, normally following a stressor event [2]. However, acute sarcopenia has been poorly characterised to date [1].

The biological mechanisms, clinical risk factors, longer term outcomes, and most effective management strategies of acute sarcopenia are currently unknown. Acute sarcopenia is considered to be caused by a combination of heightened inflammation and muscle disuse during bedrest. Studies involving healthy volunteers have demonstrated that bedrest is associated with declines in muscle quantity, strength, and aerobic performance, and that this effect is exacerbated by age [3, 4]. Acute illness (e.g. acute bacterial infection) and major surgery are associated with systemic inflammatory response [5] and endocrinological stress response (e.g. increased cortisol, decreased dehydroepiandrosterone sulfate (DHEA-s)) [6]. Pro-inflammatory cytokines activate pathways leading to increased muscle protein degradation [7] and hypercortisolaemia has been shown to exacerbate loss of muscle quantity during bedrest [8]. It has been postulated that acute sarcopenia may be partially recoverable, but may increase the risk of chronic sarcopenia over time [1]. It is proposed to be related to a combination of acute inflammatory surge and bedrest during hospitalisation [1]. Characterising acute sarcopenia will enable greater understanding of the significance of changes in clinical practice, and allow risk stratification towards targeted interventions.

EWGSOP traditionally recommended Computed Tomography $(\mathrm{CT})$, Magnetic Resonance Imaging, or Dual-Energy X-Ray Absorptiometry to measure muscle quantity [9]. However, these tests cannot be used at the bedside and have limitations when used serially [10]. Ultrasound measurement of Bilateral Anterior Thigh Thickness (BATT) has excellent inter-rater and intrarater variability [11]. EWGSOP2 supports use of ultrasound for clinical assessment of sarcopenia [2] and a consensus protocol has been proposed [12]. Bioelectrical Impedance Analysis (BIA) is an alternative non-invasive tool that provides estimates of lean mass. Muscle quantity measured by BIA has been shown to correlate with BATT [13], however, BIA is more greatly affected by fluid balance $[14,15]$. BIA is also not currently recommended for use on people with implantable cardiac devices, although research suggests this is likely to be safe [16].

Colorectal surgery is commonly performed on older adults [17]. It is not typically associated with cachexia, when performed for localised colorectal cancer [18]; metastatic cancer is known to be associated with increased risk of cachexia compared to localised cancer [19]. Colorectal surgery patients do not typically present with disease-associated pre-operative functional decline associated [20], as compared to orthopaedic or vascular surgery, where impairments in function are presenting symptoms of the illnesses themselves [21, 22]. This offers the opportunity for pre-insult measurements to be taken prior to hospitalisation. Previous studies have demonstrated acute declines in handgrip strength and muscle quantity using BIA in older adults admitted electively for colorectal surgery [23]. Acute reductions in BATT and usual gait speed were also demonstrated in our pilot study, which was used to refine this protocol [24]. Interestingly, an apparent increase in BATT was demonstrated immediately postoperatively [24]; this may be related to fluid balance but warrants further investigation [25]. However, emergency admitted patients may be at the greatest risk of declines in muscle quantity and function due to increased inflammation. Within the UK, hospitalised older adults are most commonly admitted to general medicine wards [26]. Studies involving medical and orthopaedic patients have shown variable changes in muscle quantity and function in hospitalised older adults [23, 27, 28], and changes have not been evaluated in patients admitted for emergency abdominal surgery.

\section{Methods \\ Aim}

To clinically and biologically characterise acute sarcopenia in older hospital populations, assessing for within group differences in elective colorectal surgery, emergency surgery, and general medicine patients. This will enable determination of mechanisms and identification of potential intervention strategies.

\section{Design and setting}

This is a single site cohort study at the Queen Elizabeth Hospital Birmingham (QEHB), involving 56 elective colorectal, 56 emergency abdominal surgery, and 56 medical patients. QEHB is a large tertiary hospital, with a firmly embedded research infrastructure. In the elective cohort, measurements will be performed in preoperative assessment clinic, within $48 \mathrm{~h}$ of surgery, at 7 days 
postoperatively (+/ -2 days), and at 13 weeks postoperatively (+/-1 week). In the emergency surgery cohort, we aim to recruit participants preoperatively where possible. Where this is not possible, we will recruit participants within $48 \mathrm{~h}$ of emergency surgery; further assessments will be performed at 7 days postoperatively $(+/-2$ days), and at 13 weeks postoperatively ( $+/-1$ week). Medical patients will be recruited within $48 \mathrm{~h}$ of admission with further assessment at 7 days post-admission ( $+/-2$ days), and at 13 weeks post-admission (+/-1 week). The timeframe of 13 weeks has been chosen pragmatically as a timeframe that was considered important to our patient and public involvement panel that could be feasibly conducted without high drop-out rates. The full study schema is shown in Fig. 1.

\section{Characteristics of participants}

The elective cohort will include patients expected to undergo major colorectal surgery, the emergency surgery cohort will include emergency admitted patients who have undergone or are planned to undergo emergency abdominal surgery, and the medical cohort will include emergency admitted patients with (or suspected) acute bacterial infections. Participants aged 70 years or older at time of recruitment will be included in all cohorts. Participants who are unable to provide written informed consent at time of recruitment will not be included in the elective cohort, although specific consent will be obtained for participants to remain in the study if they lose capacity, including details of any named consultee. In the emergency surgery and medical cohorts, personal or professional consultee declaration will be obtained if the participant is unable to provide written informed consent. Participants who are unable to understand verbal English, who were unable to mobilise prior to admission to hospital, or who have a life expectancy of less than 30 days will be excluded from all cohorts.

\section{Processes and interventions}

Table 1 shows the complete schedule of assessments that will be performed during this study. We describe the procedures that will be performed at each visit in further detail below.

\section{Muscle quantity assessment \\ Quadriceps ultrasound}

Rectus Femoris (RF) and Vastus Intermedius (VI) muscles in both legs will be assessed using two-dimensional

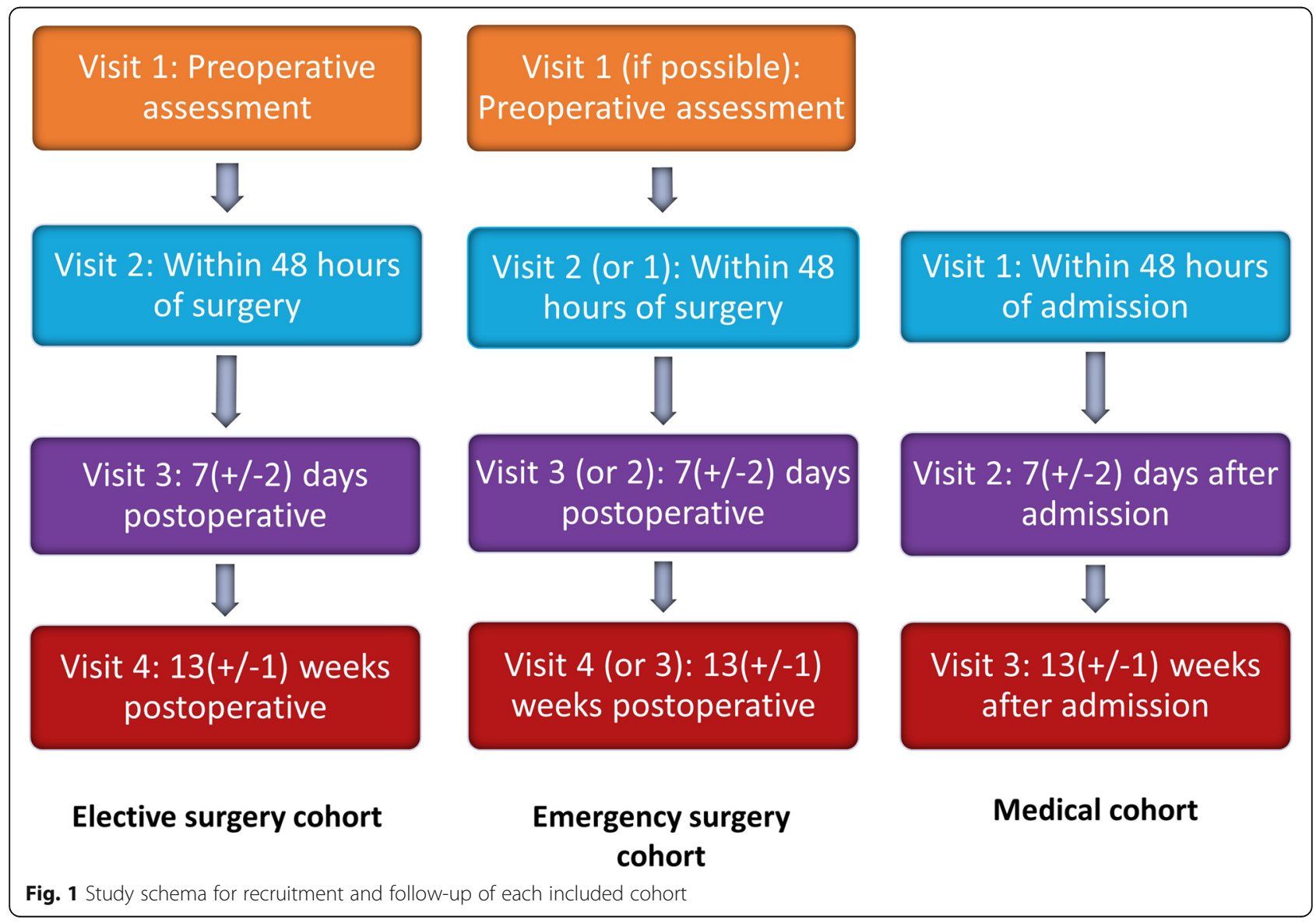


Table 1 Schedule of study procedures. This chart shows all possible visits for each cohort and assessments that would be expected to take place for each participant in each cohort. Visits marked with * may not take place for all participants

\begin{tabular}{|c|c|c|c|c|c|c|c|c|c|c|c|}
\hline \multirow[t]{2}{*}{ Visit } & \multicolumn{4}{|c|}{ Elective cohort } & \multicolumn{4}{|c|}{ Emergency cohort } & \multicolumn{3}{|c|}{ Medical cohort } \\
\hline & A & B & C & D & $A^{*}$ & B & $\mathrm{C}$ & D & B & C & $\mathrm{D}$ \\
\hline $\begin{array}{l}\text { Demographics, observations, medications, medical history, blood tests as } \\
\text { part of routine care }\end{array}$ & $\checkmark$ & $\checkmark$ & $\checkmark$ & & $\checkmark$ & $\checkmark$ & $\checkmark$ & & $\checkmark$ & $\checkmark$ & $\checkmark$ \\
\hline Review of CT scans performed as part of routine clinical care (if available) & $\checkmark$ & & & & $\checkmark$ & & & & & & \\
\hline BATT using ultrasound & $\checkmark$ & $\checkmark$ & $\checkmark$ & $\checkmark$ & $\checkmark$ & $\checkmark$ & $\checkmark$ & $\checkmark$ & $\checkmark$ & $\checkmark$ & $\checkmark$ \\
\hline BIA & $\checkmark$ & $\checkmark$ & $\checkmark$ & $\checkmark$ & $\checkmark$ & $\checkmark$ & $\checkmark$ & $\checkmark$ & $\checkmark$ & $\checkmark$ & $\checkmark$ \\
\hline Handgrip strength & $\checkmark$ & $\checkmark$ & $\checkmark$ & $\checkmark$ & $\checkmark$ & $\checkmark$ & $\checkmark$ & $\checkmark$ & $\checkmark$ & $\checkmark$ & $\checkmark$ \\
\hline Short Physical Performance Battery & $\checkmark$ & & & $\checkmark$ & & & & $\checkmark$ & $\checkmark$ & $\checkmark$ & $\checkmark$ \\
\hline Gait speed alone & & & $\checkmark$ & & & & $\checkmark$ & & & & \\
\hline Physical function by PROMIS & $\checkmark$ & & $\checkmark$ & $\checkmark$ & $\checkmark$ & & $\checkmark$ & $\checkmark$ & $\checkmark$ & $\checkmark$ & $\checkmark$ \\
\hline Katz ADLs and Lawton IADLs & $\checkmark$ & & $\checkmark$ & $\checkmark$ & $\checkmark$ & & $\checkmark$ & $\checkmark$ & $\checkmark$ & $\checkmark$ & $\checkmark$ \\
\hline Mini Nutritional Assessment (Full) & $\checkmark$ & & & $\checkmark$ & $\checkmark$ & & & $\checkmark$ & $\checkmark$ & & $\checkmark$ \\
\hline Extra frailty assessments & $\checkmark$ & & $\checkmark$ & $\checkmark$ & $\checkmark$ & & $\checkmark$ & $\checkmark$ & $\checkmark$ & $\checkmark$ & $\checkmark$ \\
\hline Venepuncture (optional) & $\checkmark$ & $\checkmark$ & & & $\checkmark$ & $\checkmark$ & & & $\checkmark$ & & \\
\hline Application of physical activity recorder (optional) & & $\checkmark$ & & & & $\checkmark$ & & & $\checkmark$ & & \\
\hline Delirium assessment & & $\checkmark$ & $\checkmark$ & & $\checkmark$ & $\checkmark$ & $\checkmark$ & & $\checkmark$ & $\checkmark$ & \\
\hline Fluid balance assessment & & $\checkmark$ & $\checkmark$ & & $\checkmark$ & $\checkmark$ & $\checkmark$ & & $\checkmark$ & $\checkmark$ & \\
\hline Participant feedback & & & & $\checkmark$ & & & & $\checkmark$ & & & $\checkmark$ \\
\hline
\end{tabular}

PROMIS Patient Reported Outcome Measures Information System, ADLs Activities of Daily Living.

Visit A: Preoperative assessment; in preoperative assessment clinic for elective cohort, on ward prior to surgery for emergency surgery cohort (where possible) not applicable to medical cohort.

Visit B: Immediate; Within $48 \mathrm{~h}$ of surgery for surgical cohorts, within $48 \mathrm{~h}$ of admission for medical cohort.

Visit C: One week; $7(+/-2)$ days after surgery (surgical cohorts) or after admission (medical cohort).

Visit D: Three months; $13(+/-1)$ week after surgery (surgical cohorts) or admission (medical cohort).

B-mode ultrasonography with a linear probe, as previously described [11]. This will be performed at first visit, immediately postoperatively (where applicable), at 7 day follow-up, and at 13 week follow-up. Participants will be positioned semi-upright with knees resting at $10-20^{\circ}$ and advised to relax their muscles. The distance from greater trochanter to knee lateral joint line will be recorded and a mark placed on the skin mid-way between the two points. Measurements will be taken in line horizontally with these marks. Contact gel will be applied. Muscle thickness will be measured with the probe in transverse position. Depth will be adjusted until the femur and overlying structures are visible. The probe will be positioned such that the widest area of the RF appears over the midpoint of the femur. Frozen images at this location will be taken with the probe held in maximal relaxation.

Thickness measurements of subcutaneous tissues (SC), $\mathrm{RF}$, and VI in a vertical line will be recorded, not including the fascia. Three frozen images will be used for all patients; a further image will be taken if there is greater than $10 \%$ variability between measurements. The mean of each reading will be used for analysis. BATT will be calculated as total thickness of right $\mathrm{VI}+$ right $\mathrm{RF}+\mathrm{left}$ $\mathrm{VI}+$ left RF. BATT: SC ratio (BATT-SCR) will be calculated as BATT divided by total thickness of right SC + left SC [24]. Where possible, cross-sectional area of the right and left RF will be measured. All measurements will be performed by an investigator with training in taking these measurements. The reliability of BATT has been shown to be excellent when using the same protocol and same machine (intraclass coefficients $>0.9$ for both intra-rater and inter-rater variability) [11].

A further image will be taken in the longitudinal position at each visit. Images will be saved and downloaded for assessment. RF and SC echogenicity will be determined using grey-scale analysis on Image J software. Pennation angle will be measured by the angle of insertion of the fascicles within the VI to the deep aponeurosis. The mean measure from up to three fascicles measured on each image will be used for analysis [29].

\section{Bioelectrical impedance analysis (BIA)}

BIA measurements will be taken at the first visit, immediately postoperatively (where applicable), at 7 day follow-up, and at 13 week follow-up using a multifrequency analyser; Bodystat Quadscan 4000. This will not be performed if the participant has an implanted permanent pacemaker or defibrillator. The participant will be positioned lying semi-upright with knees resting 
in extension on the examination couch, hospital bed or equivalent. The assessor will ensure that limbs are not touching. Two electrodes will be placed on the right foot; one below the base of the toes and the other on the ankle between the medial and lateral malleoli. The red alligator clip will be attached to the electrode nearest the toes and the black to the one at the ankle. A further two electrodes will be placed on the right hand; one behind the knuckles and the other on the wrist next to the ulnar head. The red alligator clip will be attached to the electrode nearest the fingers and the black to the one at the wrist. Electrodes will be placed transversely so that the non-stick electrode connector is facing the researcher. The Bodystat Quadscan 4000 includes a quality control feature; an impedance graph is displayed prior to results being displayed. If the graph shows a smooth curve, the investigator will proceed to record results. If there are any bumps in the graph, the investigator will recheck lead and limb position prior to repeating the analysis. Single measurements will be recorded at each visit.

All returned measures including prediction marker, impedance, resistance, reactance, phase angle, fat weight, lean weight, dry lean weight, Fat Free Mass Index (FFMI), Body Fat Mass Index (BFMI), total body water, extracellular water, and intracellular water will be recorded. Skeletal muscle mass (SMM) will be additionally estimated using three previously validated equations: 1 ) $\mathrm{SMM}=0.566 \mathrm{x}$ Fat Free Mass (lean mass) [30]; 2) $\mathrm{SMM}=\left[\left(\right.\right.$ height $^{2} / \quad$ resistance $\left.) \quad \times 0.401\right)+(\operatorname{sex} \quad \times$ $3.825)+($ age $x-0.071)]+5.102$. In the second equation, height is in $\mathrm{cm}$, for sex male $=1$, female $=0$, and age is in years [31]. 3) $\mathrm{SMM}=-3.964+\left(0.227 \times\right.$ height $^{2} / \mathrm{re}^{-}$ sistance $))+(0.095 \mathrm{x}$ weight $)+(1.384 \mathrm{x}$ sex $)+(0.064 \mathrm{x}$ reactance) [32]. For all equations, the skeletal muscle index (SMI) will be calculated through the formula $\mathrm{SMI}=\mathrm{SMM} /$ height $^{2}$, where height is in $\mathrm{m}$, for comparison with normative populations [33]. Height and weight are recorded for all patients at the site of this study as part of routine clinical care. For the elective cohort, height will be measured in preoperative assessment clinic using a stadiometer. For the emergency surgery and medical cohorts, height will be recorded using a stadiometer where possible. Where this is not possible, height will be taken from previous clinical records if these are available, or from patient report. If none of these methods are possible, then height may be estimated by measuring ulna length and conversion as per British Association of Parenteral and Enteral Nutrition guidelines [34]. If an estimate has been used, this will be recorded. The same height will be used for all visits.

L3-CT using imaging performed during routine medical care CT scans will be reviewed if these have been performed as part of routine care and are available. Skeletal muscle index will be calculated at the level of the third lumbar vertebra (L3) on the first image with both vertebral spines visible using local hospital site Picture Archiving and Communication Software. This will be calculated by manually identifying skeletal muscles and automatic calculation of cross-sectional area; this value will be corrected for height $^{2}$ [35]. Total psoas area (TPA) will also be calculated on the same slice. The right and left psoas muscle borders will be manually outlined and TPA will be calculated within the selected area. This measurement will be corrected for height ${ }^{2}$ [36]. This will be performed by the investigating geriatrician who is trained in use of the software.

\section{Muscle function assessment Muscle strength}

Handgrip strength will be measured at first visit, immediately postoperatively (where applicable), at 7 day follow-up, and at 13 week follow-up using a Jamar handheld dynamometer. Where the participant can sit in a chair, handgrip strength will be measured with the elbow flexed at $90^{\mathrm{O}}$ and the forearm supinated. If measurements are taken in the bed this will be recorded; measurements will instead be performed in the most feasible upright position. Participants will be asked to "squeeze as hard as [they] can". Handgrip strength will be measured twice on each side and the highest recording of the four measurements will be used for analysis [37].

\section{Physical performance}

The Short Physical Performance Battery (SPPB) is a standardised measure of physical performance that has been shown to be sensitive to change and provides an objective measure of physical function [38]. SPPB consists of usual gait speed, side-by-side stand, semi-tandem stand, tandem stand, and five chair stands (as quickly as they can). A total score of 12 is derived, with a lower score representing reduced physical performance. The SPPB will be measured at baseline and 3 month follow-up for the elective cohort, at 3 month follow-up for the emergency surgery cohort, and at all visits for the medical cohort. Gait speed alone will be measured at 7 day follow-up for both surgical cohorts. Gait speed will be measured by asking the participants to walk a four metre course at their "usual pace". Gait speed will not be performed at recruitment in the emergency surgery cohort as this is considered unfeasible due to pain and immediate operative recovery. Measuring chair stands at 1 week post-operatively would cause increased abdominal strain, therefore, gait speed alone will be measured at this timepoint.

Physical function - patient reported outcome measures information system (PROMIS ${ }^{\circledR}$ )

PROMIS physical function is a validated measure of physical function $[39,40]$. PROMIS is an initiative that 
compares participant responses to a reference population and derives a $\mathrm{T}$-score, where 50 is the mean, and 10 is the standard deviation. PROMIS will be measured at baseline and 3 month follow-up for all groups. The raw scores will be entered into the HealthMeasures scoring service, powered by Assessment Center ${ }^{\mathrm{SM}}$ to derive Tscores.

\section{Comprehensive geriatric assessment \\ Demographics and comorbidities}

Participant demographics, observations, medications, medical history, smoking and alcohol history, and blood test results performed as part of routine care will be collected. Medical comorbidities will be used to derive the Geriatric Index of Comorbidity [41]. Number of admissions and falls over the previous year will be recorded. Falls will be recorded from participant report. Any new information including changes in weight, observations, medications, or blood tests will be recorded at each visit, dependent on when these are recorded as part of usual clinical care.

\section{Nutritional assessment}

The Mini-Nutritional Assessment $\left(\mathrm{MNA}^{\circ}\right)$ is a validated assessment tool for nutritional status [42]. Much of the information required will be collected elsewhere. Additional information that will be collected will include food intake, specifically protein and fruit or vegetable intake, and fluid intake from participant report. Mid-arm circumference will be measured at the mid-point between the olecranon and acromium. Calf circumference will be measured as the widest part of calf. These measurements will be taken for the dominant limb. The MNA (full form) will be assessed at baseline visits and 13 week follow-up visits for each group.

\section{Frailty assessment}

Frailty will be assessed at first visit, 7 day follow-up, and 13 week follow-up using the Frailty Index (FI) [43], 9point Clinical Frailty Scale (CFS) [44], and phenotype definition [45]. Activities of Daily Living (ADLs) will be assessed using Katz (basic ADLs) [46] and Lawton (instrumental ADLs) [47] tools. The phenotypic diagnosis of frailty will be made if the participant meets three out of five criteria: low gait speed, low handgrip strength, weight loss, self-reported exhaustion or low physical activity. Cut-offs used in the original phenotype diagnosis will be used for gait speed, handgrip strength, and weight loss. Self-reported exhaustion will be defined if the participant answers "most of the time" or "all of the time" to how often over the last week they had felt that either "everything [they] did was an effort" or they "could not get going" [45]. Physical activity will be defined through self-report by asking the participant if over the last 3 months they have performed no weightbearing physical activity, been for a short walk once/ month or less, or spent more than 4 hours/ day sitting [48]. The FI will be calculated by counting the total number of deficits present out of 36 defined criteria, and dividing by 36 . These criteria have been adapted for secondary care use from those previously validated in a UK community setting to form the electronic frailty index (Supplementary File 1) [49]. The CFS will be determined by the investigating geriatrician after clinical review and after all other information has been collected. The investigating geriatrician will determine this immediately after reviewing the participant by considering ADLs, physical function, self-reported exhaustion, and symptomatic burden reported by the participant.

\section{Delirium screening and assessment}

Delirium will be diagnosed by the investigating geriatrician as per the Diagnostic and Statistical Manual of Mental Disorders 5 [50]. Participants will first be screened for evidence of delirium at each visit using the Single Question in Delirium "Do you think this patient has been more confused lately?" [51]. The investigating geriatrician will review notes and ask staff caring for the participant, family members, and the participant themselves. The participant themselves will be specifically asked "Has anything strange been happening?", such as experiencing hallucinations. Where possibility of delirium is raised upon screening or during other assessments, the investigating geriatrician will perform further assessments to formally diagnose delirium by testing attention by months of the year backwards [52], consciousness by the Modified Richmond Agitation and Sedation Scale [53], and cognition by the Abbreviated Mental Test Score (Supplementary File 2).

\section{Fluid balance assessment}

Fluid balance will be assessed and recorded during all visits during hospitalisation. Fluid balance will be assessed by clinical assessment, review of input/ output charts, and BIA measurements. Clinical assessment by the investigating geriatrician will include review of skin turgor, mucus membranes, oedema, Jugular Venous Pressure level, trends in observations e.g. blood pressure, and patient presentation. The overall fluid status will be recorded as hypovolaemic, euvolaemic, or hypervolaemic for the participant overall. However, if unilateral oedema is present in a single limb this will be recorded. BIA measurements of TBW, ECW, ICW, and third space water will be recorded separately and assessed against all other available information of fluid balance. 


\section{Other outcome data}

Further routinely collected data that will be recorded will include (as applicable) the operation performed, peri-operative blood loss, type of post-operative analgesia (patient-controlled analgesia or epidural), postoperative complications, length of stay, discharge destination, other hospital admissions within the 3 month follow-up period, histological diagnosis (cancer vs. not), and 1 year mortality.

\section{Participant feedback}

There is no standardised assessment tool for measuring test acceptability. However, a multi-faceted construct of acceptability has been proposed, which reflects the extent to which people receiving a healthcare investigation or intervention consider it to be appropriate. This construct consists of the affective attitude of the individual, procedure burden, individual ethicality (individual value system), intervention coherence (participant understanding), opportunity costs, perceived effectiveness, and selfefficacy (confidence that they can perform the necessary behaviour) [54]. Considering this construct, we have devised a questionnaire that assesses each of these aspects separately for muscle quantity (for both ultrasound and BIA), handgrip strength, and gait speed testing (Supplementary file 2). These four aspects have been chosen as these will be measured most frequently for all participants in this study and have potential for direct translation into clinical practice. This will be administered to all participants at their final visit.

\section{Venepuncture (optional)}

Blood samples will be taken using the BD vacutainer Safety-Lok $^{\mathrm{Tm}}$ system in sterile vacutainers without additives (BD biosciences). Samples will normally be taken peripherally but may be taken centrally or via arterial lines if these are in place as part of routine clinical care. Samples will be taken at first visit, and where possible, within $48 \mathrm{~h}$ of surgery in the surgical cohorts. Blood samples will be centrifuged within $30 \mathrm{~min}$ to 1 hour of collection, within the University of Birmingham Research Laboratories, within QEHB. Serum and plasma samples will be removed using calibrated pipettes and stored at $-80{ }^{\circ} \mathrm{C}$ prior to further analysis. Serum and/or plasma concentration levels of high sensitivity CReactive Protein (hsCRP), Dehydroepiandrosterone sulfate (DHEA-s), cortisol, 25-OH vitamin D, Interleukin 6 (IL-6), Tumour Necrosis Factor Alpha (TNF- $\alpha$ ), and Insulin-like Growth Factor (IGF-1) will be measured using Enzyme-Linked Immunosorbent Assays or other appropriate tests. Further additional biomarkers may be tested as appropriate. Remaining serum and/or plasma samples will be stored for use in future ethically approved research within the University of Birmingham Research Laboratories.

\section{Fitbit inspire physical activity quantification (optional)}

The Fitbit Inspire will be applied to the non-dominant wrist during hospitalisation where this is agreed by the participant or consultee. This will record activity statistics during hospitalisation including number of steps taken, distance travelled, and sedentary time. Summary statistics will be recorded for up until 30 days after hospitalisation. Participants will be advised to wear the monitor all the time. They will be supplied with a charger and advised to charge the device every 5 days when at rest, such as at night time. Position changes (e.g. sit to stand) will not be specifically recorded with this device.

\section{Statistical analysis \\ Power calculation}

The sample size for this study has been calculated by considering estimates of the precision of outcomes; $80 \%$ power and $5 \%$ significance level have been used in calculating this sample size. Allowing for $25 \%$ loss to followup from a sample size of 56 , based on a paired t-test, the following clinically important changes may be detected with a sample size of 45 in each group (all changes are powered to be bidirectional and may be identified at multiple timeframes):

- Change of 6 for t-score derived from physical function measured by PROMIS (Mean 50, SD = 10) - from baseline to 1 week and/or from baseline to 13 weeks. This is validated from previous studies [39].

- Change of $0.66 \mathrm{~cm}$ in BATT (Mean $3.6 \mathrm{~cm}, \mathrm{SD}=1.1$ $\mathrm{cm}$ ) - from baseline to 1 week and/or from baseline to 13 weeks. This is consistent with clinical change detected in our pilot study (mean loss of $0.76 \mathrm{~cm}$ ) [24].

- Change of 0.6 in skeletal muscle mass index measured using BIA (Mean 8.5, SD = 1) - from baseline to 1 week and/or from baseline to 13 weeks. This is consistent with change detected in previous studies, consistent with acute sarcopenia [28].

- Change of $6 \mathrm{~kg}$ in handgrip strength (Mean 23, $\mathrm{SD}=10$ ) - from baseline to 1 week and/or from baseline to 13 weeks. This is validated from previous studies [37].

\section{Data analysis}

Data analysis will be conducted using IBM SPSS ${ }^{\odot}$ Version 22. Results for each cohort will be analysed separately, although secondary data analysis will be conducted on all groups together. Interim analysis is planned with involvement from a patient and public involvement 
panel. Outcomes will be summarised at baseline, 7 days postoperatively, and 13 weeks postoperatively. The data analysis of the primary research question will include the following models:

- Unadjusted model with PROMIS at 13 weeks as the outcome of interest, and the secondary outcome as the covariate of interest (i.e. change in BATT, handgrip strength and/or gait speed from baseline to 7 days).

- Adjusted model with PROMIS at 13 weeks as the outcome of interest, and the secondary outcome as the covariate of interest (i.e. change in BATT, handgrip strength and/or gait speed from baseline to 7 days), with adjustment for the baseline PROMIS score, and patient demographics (e.g. age, gender).

- Model with PROMIS at 13 weeks as the outcome of interest, with adjustment for baseline PROMIS, and all secondary outcomes as covariates to establish the strength of association between those secondary outcomes and the primary outcome. This model will be used to establish which of the secondary outcomes (i.e. change in BATT, handgrip strength and/or gait speed from baseline to 7 days) is most strongly associated with change in PROMIS at 13 weeks.

Change in PROMIS rather than change in SPPB, which can be considered an objective measure of physical function, has been selected as our primary outcome for two reasons. Firstly, our patient and public involvement panel considered their own perception of their physical function to be most important. Although perception of function may differ from objective function, how function is perceived for them as individuals was considered more important. Secondly, it will only be possible to obtain true pre-hospitalisation measures of SPPB for the elective cohort. PROMIS provides a method of evaluating physical function prior to admission in the emergency cohorts. Change in SPPB will be evaluated as a secondary outcome in the elective cohort.

\section{Acute sarcopenia}

Sarcopenia will be defined as per EWGSOP2 as handgrip strength below $16 \mathrm{~kg}$ in women or below $27 \mathrm{~kg}$ in men $[2,55]$, in combination with low muscle quantity or quality. Cut-offs for low muscle quantity and quality will be evaluated comparing the cohort against reference data in healthy young adults; cut-offs for BATT of 3.85 $\mathrm{cm}$ in women and $5.44 \mathrm{~cm}$ in men have been proposed [11]. Severe sarcopenia will be defined as additional presence of low physical performance; gait speed $0.8 \mathrm{~m} / \mathrm{s}$ or less [56] or SPPB of 8 or less [57]. Acute sarcopenia will be defined as incident sarcopenia compared to baseline measurements at recruitment. The prevalence of sarcopenia will be calculated at each visit.

\section{Patient and public involvement}

Older adults have been involved in the design and development of this research. We will host further discussion groups when analysing the results (interim and final). This will be particularly valuable when determining the significance of unexpected results. The interim meeting will be of potential value in assessing if any protocol amendments are necessary. The third discussion group will also be used to co-produce the study report. The findings of this research will be disseminated to all participants and their advocates through a written summary. The participants who are enrolled in this study itself will be the best placed to assess and comment on the acceptability of the procedures used during this study. Within the study design itself, we have devised a questionnaire to derive a multi-faceted acceptability score for assessment of muscle quantity using ultrasound, handgrip strength, and walking speed. We consider that formally interviewing participants could lead to unnecessary burden, given the time they will have already dedicated to the study itself. However, any informal feedback given will be recorded to guide further research and healthcare policy. The final formal meeting that is planned with our discussion group will encompass a full evaluation of the importance of the results of this study and will be used to co-produce any protocols for future research, as well as recommendations for healthcare policy.

\section{Trial registration}

This study was registered on ClinicalTrials.gov (identifier: NCT03858192) on 28th February 2019.

\section{Discussion}

This study will fully characterise changes in muscle quantity and function in a clinical setting and will provide invaluable information to researchers, clinicians, and patients. The results of this study have potential to lead on directly to further interventional studies to counteract these changes, with particular focus on identified mechanistic associations and clinical factors to guide risk stratification. The results may also lead to direct changes in clinical practice, including the embedding of our research tools into clinical practice, and changes in policy, such as promoting early mobilisation. Providing patients and members of the public with increased knowledge on their risk of declines in muscle quantity and function, and what this is likely to mean for them, can help to empower them in their own decision making and engagement with treatment and therapy. 
We recognise that there are a number of limitations of our study. Firstly, this is a single site study, and therefore, the results may not be generalisable to the wider population. Secondly, the cohorts we have included are disease-specific. However, we consider that our results will provide proof of concept, which can be used to guide further research to increase understanding in other disease populations. As described, our study has been powered to detect within group differences in the minimally clinical important differences as derived from other studies. However, this has not been powered separately for gender and other covariates (e.g. cancer vs. not), which may affect measurements. Interim analysis has been planned and our patient and public involvement panel will be involved in the interpretation of these results. At this stage, we will review the overall progress of the study and consider if protocol amendments may be necessary.

There is some evidence that position can affect measurement of muscle quantity by ultrasound and BIA [58, 59]. However, the results will be compared to a reference group of young healthy individuals taken in the same position as we have described, using the same technique [11]. The position described is one that we can consider to be feasible for measurements in a variety of different clinical environments, whilst ensuring the quadriceps are relaxed. Particular care will be taken to standardise the position of each measure for each participant across separate visits. Nevertheless, we acknowledge that due to measurements being taken in different clinical environment, there may be small uncontrollable differences in position across visits.

Venepuncture and physical activity recording have both been included as optional aspects of the study, and it is not known what percentage of participants will agree to these. However, venepuncture was previously included as an optional aspect within our pilot study and all participants were in agreement with this [24]. We recognise that there will be limitations of physical activity measurements recorded through the Fitbit Inspire. These devices will be unable to specifically measure change in position (e.g. sit to stand). Previous studies using raw accelerometer data have shown a floor effect when measuring physical activity in frail, sedentary older adults [60]. However, physical activity measures using Fitbits have also shown to correlate well with raw accelerometer data in studies involving older adults [61]. The Fitbit Inspire is considered to be an acceptable device for older adults due to its simple wristwatch-like design, and their low cost means that they are potentially utilisable in clinical practice. Within our study, we will assess the feasibility of using these devices and assess the validity of data recorded as covariates and predictors of change in muscle parameters and physical function.
Despite these limitations, we consider the recruitment of a complex heterogeneous population to be a strength of this study. Frail older adults are frequently underrepresented in research studies. It is not possible to be certain that changes seen in young healthy adults are concordant with changes in older adults. Determining the mechanisms involved in the development of acute sarcopenia will enable risk stratification, and targeted interventions to prevent or even reverse the effects.

\section{Supplementary information}

Supplementary information accompanies this paper at https://doi.org/10. 1186/s12877-020-01626-4.

\section{Additional file 1.}

Additional file 2 .

\section{Abbreviations}

ADLs: Activities of Daily Living; CFS: Clinical Frailty Scale; cm: Centimetres; DHEA-s: Dehydroepiandrosterone sulfate; Fl: Frailty Index; hsCRP: High sensitivity C-Reactive Protein; IGF-1: Insulin-like Growth Factor 1; IL6: Interleukin 6; kg: Kilograms; L3: Third Lumbar vertebra; MNA: MiniNutritional Assessment; PROMIS: Patient Reported Outcome Measures Information System; SD: Standard Deviation; SPPB: Short Physical Performance Battery; TNF-a: Tumour Necrosis Factor Alpha; TPA: Total Psoas Area

\section{Acknowledgements}

Not applicable.

\section{Authors' contributions}

CW designed the research question and study protocol. TAJ, CAG, and TM all provided supervision to $\mathrm{CW}$ and contributed towards design of the study protocol. TP reviewed and contributed towards the study protocol. All authors read and agreed the final submitted manuscript.

\section{Authors' information}

$\mathrm{CW}$ is a senior registrar in geriatric medicine within Health Education West Midlands; she is currently registered as a PhD student at the University of Birmingham. TAJ is a clinician scientist and consultant geriatrician within the Institute of Inflammation and Ageing, University of Birmingham and University Hospitals Birmingham NHS Trust. CAG a translational scientist with research interests in the influence of use, disuse, age and disease on human skeletal muscle mass and function within the School of Sport, Exercise and Rehabilitation Sciences at the University of Birmingham. TM is a consultant geriatrician at Nottingham University Hospitals NHS Trust and honorary professor at the University of Nottingham. He is also the current president of the British Geriatrics Society. TP is a consultant colorectal surgeon at University Hospitals Birmingham NHS Trust and chair of surgical trials at the University of Birmingham.

\section{Funding}

This research has been funded by the Dowager Countess Eleanor Peel Trust (\#253) to support project costs and the Medical Research Council - Versus Arthritis Centre for Musculoskeletal Ageing Research through a PhD studentship. The funders were not involved in the design of this study and will not be involved in the conduct, analysis of results, or generation of the study report.

\section{Availability of data and materials}

Anonymised data will be available from the corresponding author upon reasonable request.

\section{Ethics approval and consent to participate}

This research has been sponsored by and reviewed by the University of Birmingham research governance team. Ethical approval has been obtained 
from Wales REC 4 (19/WA/0036) and the Health Research Authority. Written informed consent will be obtained from all participants who are considered to have capacity to consent for themselves. Written personal or professional consultee declaration will be obtained if the participant is considered to lack capacity to consent to participation.

\section{Consent for publication}

No identifiable information will be included within published material; this has been stated on all consent forms and consultee declaration forms.

\section{Competing interests}

We, the authors, confirm that we have no competing interests to disclose.

\section{Author details}

${ }^{1}$ Medical Research Council and Versus Arthritis Centre for Musculoskeletal Ageing Research, University of Birmingham and University of Nottingham, Birmingham and Nottingham, UK. ${ }^{2}$ Institute of Inflammation and Ageing, University of Birmingham, Birmingham, UK. ${ }^{3}$ University Hospitals Birmingham NHS Foundation Trust, Birmingham, UK. ${ }^{4}$ University of Birmingham Research Laboratories, Queen Elizabeth Hospital Birmingham, Mindelsohn Way, Edgbaston, Birmingham B152GW, UK. ${ }^{5}$ Birmingham Biomedical Research Centre, University of Birmingham and University Hospitals Birmingham NHS Foundation Trust, Birmingham, UK. 'School of Sport and Exercise Sciences, University of Birmingham, Birmingham, UK. ${ }^{7}$ School of Sport, Exercise and Rehabilitation Sciences, University of Birmingham, Edgbaston, Birmingham B15 2TT, UK. ${ }^{8}$ University of Nottingham, Nottingham, UK. ${ }^{9}$ Nottingham University Hospitals NHS Trust, Nottingham, UK. ${ }^{10} \mathrm{Clinical}$ Gerontology Research Unit (CGRU), First Floor, South Corridor, City Hospital, Nottingham NG5 1PB, UK. ${ }^{11}$ Academic Department of Surgery, University of Birmingham, Room 29, 4th Floor, Heritage Building, Edgbaston, Birmingham B15 2TH, UK ${ }^{12}$ University of Birmingham Research Laboratories, Queen Elizabeth Hospital Birmingham, Mindelsohn Way, Edgbaston, Birmingham B152GW, UK.

\section{Received: 4 August 2019 Accepted: 22 June 2020}

Published online: 10 July 2020

\section{References}

1. Welch C, Hassan-Smith ZK, Greig CA, Lord JM, Jackson TA. Acute sarcopenia secondary to hospitalisation - an emerging condition affecting older adults. Aging Dis. 2018;9(1):151-64.

2. Cruz-Jentoft AJ, Bahat $G$, Bauer J, Boirie $Y$, Bruyère $O$, Cederholm T, et al. Sarcopenia: revised European consensus on definition and diagnosis. Age Ageing. 2018:afy169-afy.

3. Kortebein P, Ferrando A, Lombeida J, Wolfe R, Evans WJ. Effect of 10 days of bed rest on skeletal muscle in healthy older adults. JAMA. 2007;297(16): 1772-4

4. Tanner RE, Brunker LB, Agergaard J, Barrows KM, Briggs RA, Kwon OS, et al. Age-related differences in lean mass, protein synthesis and skeletal muscle markers of proteolysis after bed rest and exercise rehabilitation. J Physiol. 2015:593(18):4259-73.

5. Smeets BJJ, Brinkman DJ, Horsten ECJ, Langius JAE, Rutten HJT, de Jonge WJ, et al. The effect of Myopenia on the inflammatory response early after colorectal surgery. Nutr Cancer. 2018;70(3):460-6.

6. Butcher SK, Killampalli V, Lascelles D, Wang K, Alpar EK, Lord JM. Raised cortisol: DHEAS ratios in the elderly after injury: potential impact upon neutrophil function and immunity. Aging Cell. 2005;4(6):319-24.

7. De Larichaudy J, Zufferli A, Serra F, Isidori AM, Naro F, Dessalle K, et al. TNFa- and tumor-induced skeletal muscle atrophy involves sphingolipid metabolism. Skelet Muscle. 2012;2(1):2.

8. Paddon-Jones D, Sheffield-Moore M, Cree MG, Hewlings SJ, Aarsland A, Wolfe RR, et al. Atrophy and impaired muscle protein synthesis during prolonged inactivity and stress. J Clin Endocrinol Metab. 2006;91(12):4836-41.

9. Cruz-Jentoft AJ, Baeyens JP, Bauer JM, Boirie Y, Cederholm T, Landi F, et al. Sarcopenia: European consensus on definition and diagnosis: report of the European working group on sarcopenia in older people. Age Ageing. 2010; 39(4):412-23.

10. Biswas DB, Jesse E, Bohan M, Simpson AK, Whang PG, Grauer JN. Radiation exposure from musculoskeletal computerized tomographic scans. J Bone Joint Surg. 2009;91(8):1882-9.
11. Wilson DV, Moorey H, Stringer H, Sahbudin I, Filer A, Lord JM, et al. Bilateral anterior thigh thickness: a new diagnostic tool for the identification of low muscle mass? J Am Med Dir Assoc. 2019.

12. Perkisas S, Baudry S, Bauer J, Beckwée D, De Cock A-M, Hobbelen H, et al. Application of ultrasound for muscle assessment in sarcopenia: towards standardized measurements. Eur Geriatr Med. 2018.

13. Wilson D. Frailty, sarcopenia and immunesenescence: shared mechanisms and clinical insights [PhD]: University of Birmingham; 2018.

14. Ticinesi A, Meschi T, Narici MV, Lauretani F, Maggio M. Muscle ultrasound and sarcopenia in older individuals: a clinical perspective. J Am Med Dir Assoc. 2017:18(4):290-300.

15. Nakanishi N, Tsutsumi R, Okayama Y, Takashima T, Ueno $Y$, Itagaki T, et al. Monitoring of muscle mass in critically ill patients: comparison of ultrasound and two bioelectrical impedance analysis devices. J Intensive Care. 2019; $7(1): 61$.

16. Chabin X, Taghli-Lamallem O, Mulliez A, Bordachar P, Jean F, Futier E, et al. Bioimpedance analysis is safe in patients with implanted cardiac electronic devices. Clin Nutr (Edinburgh, Scotland). 2019;38(2):806-11.

17. The Royal College of Surgeons of England. Access All Ages: Assessing the impact of ages on access to surgical treatment. 2012.

18. Fox KM, Brooks JM, Gandra SR, Markus R, Chiou C-F. Estimation of Cachexia among Cancer Patients Based on Four Definitions. J Oncol. 2009;2009: 693458.

19. Shiono M, Huang K, Downey RJ, Consul N, Villanueva N, Beck K, et al. An analysis of the relationship between metastases and cachexia in lung cancer patients. Cancer Med. 2016:5(9):2641-8.

20. Smith D, Ballal M, Hodder R, Soin G, Selvachandran SN, Cade D. Symptomatic presentation of early colorectal cancer. Ann R Coll Surg Engl. 2006;88(2):185-90.

21. Regensteiner JG, Hargarten ME, Rutherford RB, Hiatt WR. Functional benefits of peripheral vascular bypass surgery for patients with intermittent claudication. Angiology. 1993;44(1):1-10.

22. Kapstad H, Rustøen T, Hanestad BR, Moum T, Langeland N, Stavem K. Changes in pain, stiffness and physical function in patients with osteoarthritis waiting for hip or knee joint replacement surgery. Osteoarthr Cartil. 2007:15(7):837-43.

23. Van Ancum JM, Scheerman $\mathrm{K}$, Jonkman NH, Smeenk HE, Kruizinga RC, Meskers CGM, et al. Change in muscle strength and muscle mass in older hospitalized patients: a systematic review and meta-analysis. Exp Gerontol. 2017:92:34-41.

24. Welch C, Greig CA, Hassan-Smith ZK, Pinkney T, Lord JM, Jackson TA. A pilot observational study measuring acute sarcopenia in older colorectal surgery patients; 2018.

25. Fischer A, Spiegl M, Altmann K, Winkler A, Salamon A, Themessl-Huber M, et al. Muscle mass, strength and functional outcomes in critically ill patients after cardiothoracic surgery: does neuromuscular electrical stimulation help? The Catastim 2 randomized controlled trial. Crit Care. 2016:20:30.

26. Digital NHS. Hospital admitted patient care activity 2018-19; 2019.

27. Van Ancum JM, Scheerman K, Pierik VD, Numans ST, Verlaan S, Smeenk HE, et al. Muscle strength and muscle mass in older patients during hospitalization: the EMPOWER study. Gerontology. 2017:63(6):507-14.

28. Martone AM, Bianchi L, Abete P, Bellelli G, Bo M, Cherubini A, et al. The incidence of sarcopenia among hospitalized older patients: results from the glisten study. J Cachexia Sarcopenia Muscle. 2017.

29. Strasser EM, Draskovits T, Praschak M, Quittan M, Graf A. Association between ultrasound measurements of muscle thickness, pennation angle, echogenicity and skeletal muscle strength in the elderly. Age (Dordr). 2013; 35(6):2377-88

30. Bahat G, Tufan A, Tufan F, Kilic C, Akpinar TS, Kose M, et al. Cut-off points to identify sarcopenia according to European Working Group on Sarcopenia in Older People (EWGSOP) definition. Clinical nutrition (Edinburgh, Scotland). 2016;35(6):1557-63.

31. Janssen I, Heymsfield SB, Baumgartner RN, Ross R. Estimation of skeletal muscle mass by bioelectrical impedance analysis. J Appl Physiol. 2000;89(2): 465-71.

32. Sergi G, De Rui M, Veronese N, Bolzetta F, Berton L, Carraro S, et al. Assessing appendicular skeletal muscle mass with bioelectrical impedance analysis in free-living Caucasian older adults. Clin Nutr. 2015;34(4):667-73.

33. Janssen I, Baumgartner RN, Ross R, Rosenberg $\Vdash H$, Roubenoff R. Skeletal muscle cutpoints associated with elevated physical disability risk in older men and women. Am J Epidemiol. 2004;159(4):413-21. 
34. British Association of Parenteral and Enteral Nutrition. Malnutrition Universal Screening Tool. 2011.

35. van der Werf A, Langius JAE, de van der Schueren MAE, Nurmohamed SA, van der Pant KAMI, Blauwhoff-Buskermolen S, et al. Percentiles for skeletal muscle index, area and radiation attenuation based on computed tomography imaging in a healthy Caucasian population. Eur J Clin Nutr. 2018;72(2):288-96

36. Hervochon R, Bobbio A, Guinet C, Mansuet-Lupo A, Rabbat A, Régnard J-F, et al. Body mass index and Total psoas area affect outcomes in patients undergoing Pneumonectomy for Cancer. Ann Thorac Surg. 2017;103(1):287-95.

37. Roberts HC, Denison HJ, Martin HJ, Patel HP, Syddall H, Cooper C, et al. A review of the measurement of grip strength in clinical and epidemiological studies: towards a standardised approach. Age Ageing. 2011;40(4):423-9.

38. Guralnik JM, Simonsick EM, Ferrucci L, Glynn RJ, Berkman LF, Blazer DG, et al. A short physical performance battery assessing lower extremity function: association with self-reported disability and prediction of mortality and nursing home admission. J Gerontol. 1994:49(2):M85-94.

39. Yost KJ, Eton DT, Garcia SF, Cella D. Minimally important differences were estimated for six patient-reported outcomes measurement information system-Cancer scales in advanced-stage cancer patients. J Clin Epidemiol. 2011;64(5):507-16.

40. Tatsuoka C, DeMarco L, Smyth KA, Wilkes S, Howland M, Lerner AJ, et al. Evaluating PROMIS physical function measures in older adults at risk for Alzheimer's disease. Gerontol Geriatr Med. 2016;2:2333721416665502.

41. Zekry D, Loures Valle BH, Lardi C, Graf C, Michel J-P, Gold G, et al. Geriatrics index of comorbidity was the most accurate predictor of death in geriatric hospital among six comorbidity scores. J Clin Epidemiol. 2010;63(9):1036-44.

42. Vellas B, Guigoz Y, Garry PJ, Nourhashemi F, Bennahum D, Lauque S, et al. The mini nutritional assessment (MNA) and its use in grading the nutritional state of elderly patients. Nutrition. 1999;15(2):116-22.

43. Rockwood K, Mitnitski A. Frailty in relation to the accumulation of deficits. J Gerontol A. 2007;62(7):722-7.

44. Rockwood K, Song X, MacKnight C, Bergman H, Hogan DB, McDowell I, et al. A global clinical measure of fitness and frailty in elderly people. CMAJ. 2005;173(5):489-95.

45. Fried LP, Tangen CM, Walston J, Newman AB, Hirsch C, Gottdiener J, et al. Frailty in older adults: evidence for a phenotype. J Gerontol A Biol Sci Med Sci. 2001;56(3):M146-56.

46. Katz S, Ford AB, Moskowitz RW, Jackson BA, Jaffe MW. Studies of illness in the aged. The index of ADL: a standardized measure of biological and psychosocial function. JAMA. 1963;185:914-9.

47. Lawton MP, Brody EM. Assessment of older people: self-maintaining and instrumental activities of daily living. Gerontologist. 1969;9(3):179-86.

48. Fairhall N, Aggar C, Kurrle SE, Sherrington C, Lord S, Lockwood K, et al. Frailty intervention trial (FIT). BMC Geriatr. 2008;8(1):27.

49. Clegg A, Bates C, Young J, Ryan R, Nichols L, Ann Teale E, et al. Development and validation of an electronic frailty index using routine primary care electronic health record data. Age Ageing. 2016;45(3):353-60.

50. American Psychiatric Association. Diagnostic and Statistical Manual of Mental Disorders (DSM-5 $\left.{ }^{\circledR}\right) .5$ ed2013.

51. Sands MB, Dantoc BP, Hartshorn A, Ryan CJ, Lujic S. Single question in delirium (SQiD): testing its efficacy against psychiatrist interview, the confusion assessment method and the memorial delirium assessment scale. Palliat Med. 2010;24(6):561-5.

52. Meagher J, Leonard M, Donoghue L, O'Regan N, Timmons S, Exton C, et al. Months backward test: a review of its use in clinical studies. World J Psychiatry. 2015;5(3):305-14.

53. Sessler CN, Gosnell MS, Grap MJ, Brophy GM, O'Neal PV, Keane KA, et al. The Richmond agitation-sedation scale. Am J Respir Crit Care Med. 2002;166(10): 1338-44.

54. Sekhon M, Cartwright M, Francis JJ. Acceptability of healthcare interventions: an overview of reviews and development of a theoretical framework. BMC Health Serv Res. 2017;17(1):88.

55. Dodds RM, Syddall HE, Cooper R, Benzeval M, Deary IJ, Dennison EM, et al. Grip strength across the life course: normative data from twelve British studies. PLoS One. 2014;9(12):e113637.

56. Studenski S, Perera S, Patel K, Rosano C, Faulkner K, Inzitari M, et al. Gait speed and survival in older adults. Jama. 2011;305(1):50-8.

57. Pavasini R, Guralnik J, Brown JC, di Bari M, Cesari M, Landi F, et al. Short physical performance battery and all-cause mortality: systematic review and meta-analysis. BMC Med. 2016;14(1):215.
58. Hacker ED, Peters T, Garkova M. Ultrasound assessment of the rectus Femoris cross-sectional area:subject position implications. West J Nurs Res. 2016;38(9):1221-30.

59. Slinde F, Bark A, Jansson J, Rossander-HulthÉN L. Bioelectrical impedance variation in healthy subjects during $12 \mathrm{~h}$ in the supine position. Clin Nutr. 2003;22(2):153-7.

60. Heesch KC, Hill RL, Aguilar-Farias N, van Uffelen JGZ, Pavey T. Validity of objective methods for measuring sedentary behaviour in older adults: a systematic review. Int J Behav Nutr Phys Act. 2018;15(1):119.

61. Straiton N, Alharbi M, Bauman A, Neubeck L, Gullick J, Bhindi R, et al. The validity and reliability of consumer-grade activity trackers in older, community-dwelling adults: a systematic review. Maturitas. 2018;112:85-93.

\section{Publisher's Note}

Springer Nature remains neutral with regard to jurisdictional claims in published maps and institutional affiliations.
Ready to submit your research? Choose BMC and benefit from:

- fast, convenient online submission

- thorough peer review by experienced researchers in your field

- rapid publication on acceptance

- support for research data, including large and complex data types

- gold Open Access which fosters wider collaboration and increased citations

- maximum visibility for your research: over $100 \mathrm{M}$ website views per year

At BMC, research is always in progress.

Learn more biomedcentral.com/submissions 